Revista de Biología Marina y Oceanografía

Vol. 48, №3: 591-600, diciembre 2013

DOI 10.4067/S0718-19572013000300015

Artículo

\title{
Incorporación de nitrato por fitoplancton en dos ambientes marinos diferentes de la provincia de Chubut $\left(42^{\circ}-46^{\circ} \mathrm{S}\right.$, Argentina)
}

\author{
Uptake of nitrate by phytoplankton in two different marine environments \\ in the province of Chubut $\left(42^{\circ}-46^{\circ} \mathrm{S}\right.$, Argentina) \\ Flavio E. Paparazzo', Laura Farias ${ }^{2,3}$, Norma Santinelli ${ }^{4}$, \\ Viviana A. Alder ${ }^{5,6}$ y José L. Esteves ${ }^{1}$
}

\begin{abstract}
${ }^{1}$ Centro Nacional Patagónico, CENPAT-CONICET, Blvd. Brown 2915, U9120ACD. Puerto Madryn, Chubut, Argentina. paparazzo@cenpat.edu.ar

${ }^{2}$ Departamento de Oceanografía, Facultad de Ciencias Naturales y Oceanográficas. Casilla 160-C, Universidad de Concepción, Chile ${ }^{3}$ Centro de Ciencia del Clima y la Resiliencia (CR)2, Blanco Encalada 2002, piso 4. Facultad de Ciencias Físicas y Matemáticas, Universidad de Chile, Santiago, Chile

${ }^{4}$ Universidad Nacional de la Patagonia San Juan Bosco (UNPSJB), Facultad de Ciencias Naturales, Belgrano 504, $2^{\circ}$ Piso, Trelew, Chubut, Argentina

${ }^{5}$ Instituto Antártico Argentino, IAA-DNA, Cerrito 1248, C1010AAZ, Buenos Aires, Argentina

${ }^{6}$ Departamento de Ecología, Genética y Evolución, IEGEBA-UBA-CONICET, Facultad de Ciencias Exactas y Naturales, Universidad de Buenos Aires, Int. Güiraldes 2620, C1428 EHA, Buenos Aires, Argentina
\end{abstract}

\begin{abstract}
The spatio-temporal distribution of nutrients and primary production processes are fundamental to understand the functioning of marine ecosystems. In this regard, the quantification of the new and regenerated primary production is highly relevant. In this work, the new production was estimated from the measurement of the uptake of nitrate by the phytoplankton by incubating samples enriched with stable isotopes of nitrogen $\left({ }^{15} \mathrm{NO}_{3}\right)$ together with the measurement of temperature, salinity, $\mathrm{pH}$, chlorophyll-a and the measure of the cell abundance of the major phytoplankton groups. The experiments were carried out in cape Dos Bahías (CBD) $\left(46^{\circ} \mathrm{S}\right)$ (open water environment without anthropogenic influence and depths greater than $50 \mathrm{~m}$ ) and Bahía Nueva (BN) $\left(42^{\circ} \mathrm{S}\right)$ (semi-enclosed sea environment with possible anthropogenic influence and depths less than $10 \mathrm{~m}$ ). Temperature, salinity and $\mathrm{pH}$ were similar in the 2 analyzed ecosystems. The average concentration of nitrate, phosphate and silicate in $\mathrm{BN}$ was $0.19 \pm 0.12 \mu \mathrm{M}, 0.85 \pm 0.09 \mu \mathrm{M}$ and $1.77 \pm 0.23 \mu \mathrm{M}$ respectively. Comparatively, nitrate and silicate concentration in CDB was higher $(0.86 \pm 1.28 \mu \mathrm{M}$ and $3.69 \pm 1.07 \mu \mathrm{M}$ respectively), while the phosphate concentration showed lower values $(0.66 \pm 0.20 \mu \mathrm{M})$. The concentration of chlorophyll-a was not associated with the nitrate uptake. In CBD, dinoflagellates and cryptophytes were the dominant groups and their abundance was positively correlated with the uptake of nitrate. In BN the diatoms dominated and were not associated with this parameter. We conclude that the new production in surface waters was similar for both environments and higher than in the subsurface layer.
\end{abstract}

Key words: New production, phytoplankton, chlorophyll-a, stable isotopes, inorganic nutrients, Argentina

Resumen.- La distribución espacio-temporal de los nutrientes y procesos de producción primaria son fundamentales para la comprensión del funcionamiento de ecosistemas marinos. Al respecto, adquiere gran relevancia ecosistémica la cuantificación de la producción primaria nueva y regenerada. En el presente trabajo se estimó la producción nueva a partir de la medición de la incorporación de nitrato por el fitoplancton, mediante incubación de muestras enriquecidas con isótopos estables del nitrógeno $\left({ }^{15} \mathrm{NO}_{3}\right)$ junto con la medición de temperatura, salinidad, $\mathrm{pH}, \mathrm{clorofila-a}$ y abundancia de los principales grupos fitoplanctónicos. Los experimentos fueron realizados en cabo Dos Bahías (CDB) (46 $\left.{ }^{\circ} \mathrm{S}\right)$ (ambiente de aguas abiertas sin influencia antrópica y profundidades mayores a $50 \mathrm{~m}$ ) y Bahía Nueva (BN) (42 $\left.{ }^{\circ} \mathrm{S}\right)($ ambiente de aguas semicerradas con posible influencia antrópica y profundidades menores a $10 \mathrm{~m}$ ). La temperatura, la salinidad y el pH fueron similares en los 2 ecosistemas analizados. En BN la concentración media de nitrato, fosfato y silicato fue de 0,19 $\pm 0,12 \mu \mathrm{M}, 0,85 \pm 0,09 \mu \mathrm{M}$ y 1,77 $\pm 0,23 \mu \mathrm{M}$ respectivamente. Comparativamente, en CDB la concentración de nitrato y silicato fue mayor $(0,86 \pm 1,28 \mu \mathrm{M}$ y 3,69 $\pm 1,07 \mu \mathrm{M}$ respectivamente), mientras que la concentración de fosfato presentó valores menores $(0,66 \pm 0,20 \mu \mathrm{M})$. La concentración de clorofila-a no estuvo asociada a la concentración de nutrientes. En CDB, los dinoflagelados y las criptófitas fueron los grupos dominantes y su abundancia correlacionó positivamente con la incorporación de nitrato. En BN dominaron las diatomeas, sin asociarse a esta variable. Se concluye que en aguas superficiales, la producción nueva fue similar en los 2 ambientes y mayor que en el estrato sub-superficial.

Palabras clave: Producción nueva, fitoplancton, clorofila-a, isótopos estables, nutrientes inorgánicos, Argentina 


\section{INTRODUCCIÓN}

En los ambientes acuáticos, el proceso de asimilación de nitrógeno es ejercido por el fitoplancton y bacterioplancton, principalmente en la capa eufótica. La conversión de nitrógeno inorgánico a orgánico es uno de los procesos biogeoquímicos más relevantes del ambiente asociados al fitoplancton (Cabrita et al. 1999). En aguas superficiales, Dugdale \& Goering (1967) consideraron que existen diversas fuentes nitrogenadas involucradas en la incorporación/asimilación del fitoplancton. Por un lado, el nitrógeno regenerado (principalmente como amonio) que responde a fuentes principalmente autóctonas producto de la mineralización de la materia orgánica dentro de la zona eufótica. Esta fuente no produce excedentes, todo lo que se produce se recicla. Por otro lado, se encuentra el nitrógeno nuevo (principalmente como nitrato) cuyo origen es alóctono e ingresa a la capa eufótica por advección o difusión turbulenta desde las capas más profundas del océano. Si bien la fijación de $\mathrm{N}_{2}$ es una fuente de nitrógeno nuevo y en océanos oligotróficos sustenta gran parte de la producción primaria (Montoya et al. 2004, Fernandez et al. 2011), ésta es insignificante en plataformas continentales y áreas costeras marinas. El modelo de Dugdale \& Goering (1967) restructuró el conocimiento del ciclo del nitrógeno en los océanos y además sirvió para comparar la importancia relativa de la productividad primaria sostenida por nitrato o por amonio, procesos que se conocen como producción nueva y producción regenerada respectivamente. En base a lo detallado anteriormente, se evidencia la importancia que tiene el conocimiento sobre la calidad, disponibilidad y distribución espacio-temporal de los nutrientes en los ecosistemas acuáticos para entender múltiples procesos asociados a los productores primarios en particular y a la productividad biológica marina en general. Son determinantes en los ciclos biogeoquímicos del carbono y nitrógeno, la producción exportada, formación de sedimentos biogénicos, y por ende el Cambio Climático, entre otros. Pese a la reconocida importancia del tema y de casi medio siglo de estudios intensivos, hay aún importantes regiones marinas prácticamente inexploradas. Es el caso del Mar Argentino que, si bien existen algunos estudios particulares (e.g., Brandhorst \& Castello 1971, Carreto et al. 1995, 2007; Paparazzo 2003, Paparazzo et al. 2010), prácticamente se desconoce la distribución espacio-temporal de los nutrientes y procesos asociados al reciclaje de nitrógeno y carbono. Del mismo modo, hasta el presente no se han realizado estudios experimentales que permitan conocer las magnitudes de la producción nueva, basada en nitrato.
El objetivo de este trabajo fue cuantificar y comparar la tasa de incorporación de nitrato por organismos fitoplanctónicos de un ecosistema semicerrado costero (Bahía Nueva) y uno abierto de aguas típicas de plataforma (cabo Dos Bahías), mediante la aplicación de técnicas basadas en incubaciones con isótopos estables del nitrógeno. Esos resultados fueron contrastados con la abundancia de los principales grupos fitoplanctónicos. Dado que se trata de 2 ambientes diferentes, se propone como hipótesis que la tasa de incorporación de nitrato por el fitoplancton del ecosistema de $\mathrm{BN}$ es diferente a la de CDB. El estudio constituye el primer análisis para la zona costera de la Provincia del Chubut (Argentina).

\section{MATERIALES Y MÉTOdOS}

Se realizaron muestreos de agua de mar para la medición de variables ambientales y experimentos en 2 sitios seleccionados del Mar Argentino, ubicados en la Provincia de Chubut (Fig. 1). El primer sitio investigado fue cabo Dos Bahías (CDB), cerca de la ciudad de Camarones, cuyos muestreos se realizaron durante el 6 y 7 de marzo de 2009. Se establecieron 5 estaciones oceanográficas en la columna de agua (distanciadas cada $8 \mathrm{~km}$ ), en una transecta perpendicular a la línea de costa sobre los

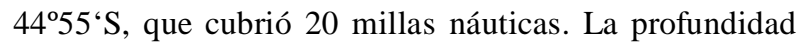
del fondo varió entre 80 y 120 m. En cada una de las estaciones se llevó a cabo un muestreo vertical para medida de salinidad y nutrientes en 3 profundidades diferentes $(0,10$ y $20 \mathrm{~m})$. Además, se realizaron mediciones de turbidez, temperatura y $\mathrm{pH}$. En las estaciones 2 y 4 se realizó, la toma de muestras para el análisis de clorofila- $a$, recuento celular de los principales grupos fitoplanctónicos y los experimentos de incorporacion de nitrato por fitoplancton a 2 profundidades (0 y $10 \mathrm{~m})$.

El segundo sitio investigado fue Bahía Nueva (BN), cercana a la ciudad de Puerto Madryn. Se establecieron 2 estaciones (16 y 17 de abril de 2009) en aguas someras (profundidad del fondo: $4 \mathrm{~m}$ ), cuya posición se seleccionó considerando a priori diferentes grados de influencia antrópica. La primera estación se ubicó $5 \mathrm{~km}$ al sudeste de la ciudad de Puerto Madryn a $100 \mathrm{~m}$ de la costa $\left(42^{\circ} \mathrm{S}\right.$, $\left.65,0^{\circ} \mathrm{O}\right)$, mientras que la segunda fue seleccionada frente a la Ciudad, a $200 \mathrm{~m}$ de la costa $\left(42^{\circ} \mathrm{S}, 64,5^{\circ} \mathrm{O}\right)$. En ambas se midieron las mismas variables que en las estaciones 2 y 4 de CDB.

Las muestras de agua de mar fueron colectadas mediante una botella Niskin. Para el análisis de nutrientes las muestras fueron filtradas por filtros GF/F y 


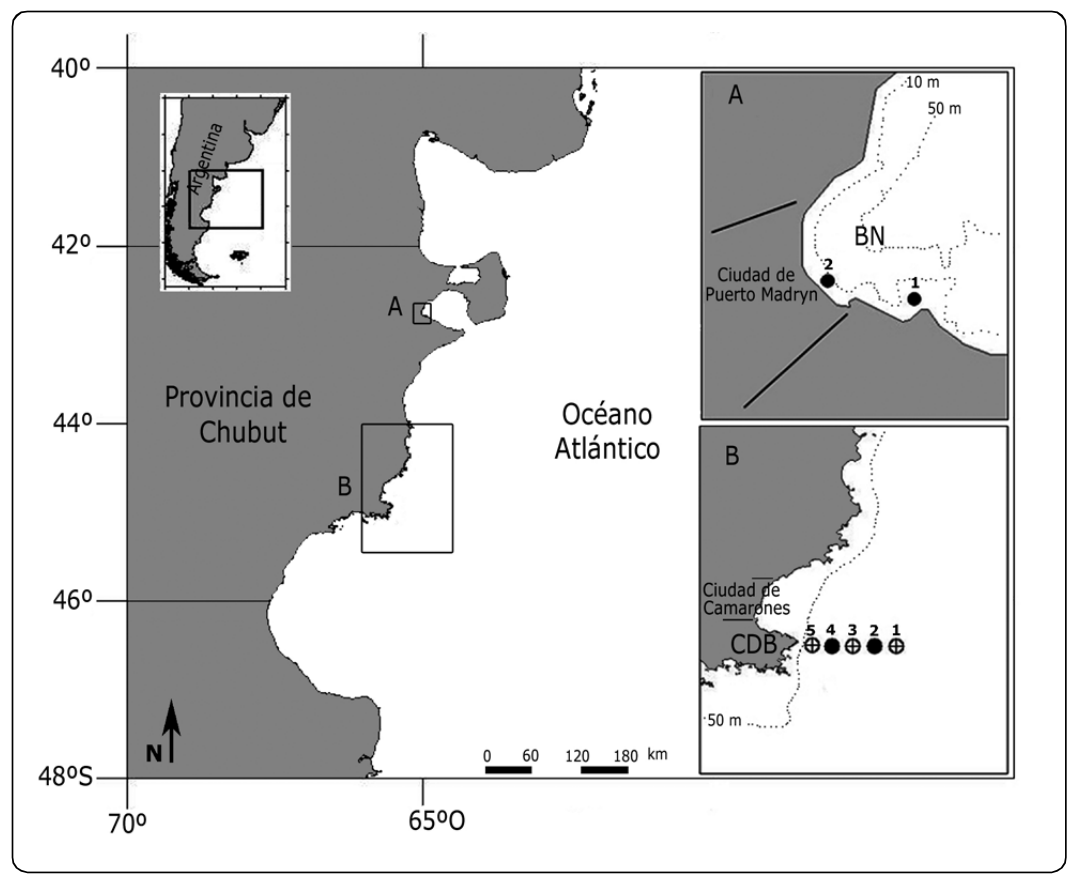

Figura 1. Ubicación geográfica de los sitios investigados en inmediaciones de (A) Bahía Nueva (BN), durante abril de 2009 y (B) cabo Dos Bahías (CBD), durante marzo de 2009. En todas las estaciones se midió temperatura, salinidad, pH, nitrato, fosfato y silicato. Los círculos negros indican las estaciones en las que, además, se midió clorofila-a, abundancia de fitoplancton y se realizaron experimentos de incorporación de nitrato / Geographic location of the sites investigated in the vicinity of (A) Bahía Nueva (BN), during April 2009 and (B) cape Dos Bahías (CBD), during March 2009. Temperature, salinity, pH, nitrate, phosphate and silicate were measured in all stations. Black circles indicate the stations where chlorophyll-a concentration and phytoplankton abundance were also measured and the uptake of nitrate determined

almacenadas en recipientes de polietileno de $250 \mathrm{ml}$, preservadas a $-20^{\circ} \mathrm{C}$ hasta el momento de su análisis realizado en laboratorio. Los recipientes fueron previamente lavados con detergente neutro, agua destilada y $\mathrm{HCl} 5 \%$ y enjuagados con agua destilada. Nitrato, nitrito y fosfato fueron medidos siguiendo las técnicas propuestas por Strickland \& Parsons (1972), con modificaciones para su análisis en autoanalizador Technicon ${ }^{\circledR}$ II. Dada la baja concentración de nitrito, se expresó la concentración de nitrito + nitrato como nitrato. El silicato fue medido siguiendo el método propuesto por Technicon (1977). La concentración de clorofila- $a$ se estimó a partir del filtrado de aproximadamente $1,5 \mathrm{~L}$ de agua de mar por filtros Whatman ${ }^{\circledR} \mathrm{GF} / \mathrm{F}$ preservados a $-20^{\circ} \mathrm{C}$ hasta su posterior análisis. La extracción fue realizada con acetona $90 \%$ y su concentración medida por fluorometría (Strickland \& Parsons 1972). La salinidad se midió por inductometría mediante salinómetro Portsal ${ }^{\circledR}$, sobre $250 \mathrm{ml}$ de agua de mar tomadas con botellas de vidrio con doble tapa. La turbidez fue medida con un disco de Secchi; la temperatura y el $\mathrm{pH}$ con un pHmetro Yokogawa ${ }^{\circledast}$ PH82.
Para cuantificar la tasa de incorporación de nitrato por fitoplancton, se realizó la puesta a punto de la técnica de incubación de agua de mar enriquecida con isótopos estables de Dugdale \& Goering (1967). Se colectaron, por duplicado, muestras de agua de mar $(600 \mathrm{ml})$, que fueron enriquecidas con $\mathrm{Na}^{15} \mathrm{NO}_{3}\left(98 \%{ }^{15} \mathrm{~N}\right.$ ) (Sigma-Aldrich ${ }^{\oplus}$ ) y puestas a incubar en botellas de $600 \mathrm{ml}$ de PVC transparente durante $24 \mathrm{~h}$. Dado que la concentración de nitrato del medio (medida un día antes del experimento) fue muy baja, la concentración del isótopo inoculada fue la mínima para la obtención de señal espectrofotométrica $(0,1 \mu \mathrm{M})$. Por medio de un incubador de red flotante diseñado específicamente para la experiencia, las muestras se mantuvieron bajo condiciones de temperatura e irradiación in situ. Para representar las condiciones de luz incidente a $3,5 \mathrm{~m}$ en BN y $10 \mathrm{~m}$ en CDB, las botellas fueron cubiertas con filtros de tela Cole Palmer ${ }^{\circledR}$ que restaron un 25\% de la irradiación recibida. Posteriormente, el volumen de cada muestra se filtró a través de filtros MGF de $0,7 \mu \mathrm{m}$ de tamaño de poro y $25 \mathrm{~mm}$ de diámetro, precombustionados a $450^{\circ} \mathrm{C}$ durante $4 \mathrm{~h}$. Luego del filtrado, los filtros se secaron en estufa a $60^{\circ} \mathrm{C}$ durante $48 \mathrm{~h} \mathrm{y}$ prensados hasta su análisis. 
La razón isotópica ${ }^{15} \mathrm{~N} /{ }^{14} \mathrm{~N}$ se obtuvo por espectrometría de masa de razón isotópica IRMS (Analizador Elemental: Thermo Finnigan ${ }^{\circledR}$ Flash EA 1112 acoplado al Espectrómetro de Masa: Thermo Finnigan ${ }^{\circledR}$ Delta Plus). De manera simultánea, se midió la concentración de Carbono Orgánico Particulado (COP) y Nitrógeno Orgánico Particulado (NOP). Previo a esto, los filtros fueron pesados para la obtención de una buena señal cromatográfica. Para la eliminación de las fracciones de carbonatos presentes en las muestras se procedió a su acidificación por medio de $\mathrm{HCl}$ fumante $12 \mathrm{~N}$ por un periodo de $8 \mathrm{~h}$ para su posterior secado.

Las muestras de fitoplancton se tomaron con botella Niskin, se conservaron en recipientes de vidrio color caramelo y se fijaron con solución de lugol. Los análisis cuantitativos se realizaron con microscopio invertido Leitz $^{\circledR}$ DMIL, siguiendo la técnica de Ütermohl (Lund et al. 1958) y las recomendaciones de Villafañe et al. (1995).

La comparación entre las medias de los parámetros analizados en diferentes estaciones de muestreo fue testeada mediante una prueba $t$ de Student (con un nivel de significación estadística de 0,05 ) y la correlación entre diferentes variables fue establecida mediante un test de Pearson utilizando el programa Statistica 7 (StatSoft Inc.)

\section{Resultados}

\section{Aguas de Plataforma: cabo Dos Bahías (CDB)}

\section{TASA DE INCORPORACIÓN DE NITRATO}

La temperatura del sector CBD (Fig. 1) fue un grado más baja en la estación más cercana a la costa (entre $15^{\circ}$ y $16^{\circ} \mathrm{C}$ ) que en las restantes (entre $16^{\circ}$ y $17^{\circ} \mathrm{C}$ ). La salinidad y el $\mathrm{pH}$ se mantuvieron relativamente constantes $(33,47 \pm$ 0,15 y $8,12 \pm 0,05$, respectivamente). Los niveles de turbidez y la concentración de nitrato y fosfato fueron significativamente más elevados en las 3 estaciones más cercanas a la costa $(P<0,05)$; en el caso del fosfato, alcanzó concentraciones casi 2 veces superiores en las de la estación más costera (del orden de $1 \mu \mathrm{M}$ ), mientras que la concentración de nitrato fue cercana a cero en las estaciones más alejadas y del orden de $3 \mu \mathrm{M}$ en las más costeras. El silicato no mostró una tendencia definida, presentó una concentración mínima de $1,76 \mu \mathrm{M}$ en aguas superficiales de la estación 3, alcanzando como máximo los 5,43 $\mu \mathrm{M}$ (Fig. 2).

Las restantes variables fueron medidas en las estaciones 2 y 4 . La clorofila- $a$ fue más elevada en el estrato superficial de la estación $2\left(1,03 \mu \mathrm{g} \mathrm{L^{-1 }}\right)$ y en el más profundo de la estación $4\left(1,45 \mu \mathrm{g} \mathrm{L}^{-1}\right)$. La tasa de incorporación de nitrato para ambas estaciones fue muy similar en el estrato superficial $\left(38 \pm 1,5 \mathrm{nM} \mathrm{d}^{-1}\right.$ y $36 \pm 3,4$ $\left.\mathrm{nM} \mathrm{d}^{-1}\right)$. Sin embargo, a $10 \mathrm{~m}$ de profundidad en la estación $4\left(60 \pm 1,2 \mathrm{nM} \mathrm{d}^{-1}\right)$ se duplicó el valor de la tasa de la estación 2 (29 $\left.\pm 3,5 \mathrm{nM} \mathrm{d}^{-1}\right)$ (Fig. 2). El COP y el NOP siguieron una tendencia similar a la tasa de incorporación de nitrato, con valores similares en superficie y a $10 \mathrm{~m}$ de profundidad (del orden de $200 \mu \mathrm{g}$ C y $22 \mu \mathrm{g} \mathrm{N}$ ). La relación COP/NOP se mantuvo entre 8,5 y 10,2. La relación $\mathrm{NO}_{3} /$ $\mathrm{PO}_{4}$ varió entre 0,1 y 1,6 , mientras que la relación $\mathrm{NO}_{3} /$ $\mathrm{SiO}_{3}$ entre 0,01 y 0,28 y la relación $\mathrm{PO}_{4} / \mathrm{SiO}_{3}$ entre 0,10 y 0,17 .

\section{DOMINANCIA DE GRUPOS FITOPLANCTÓNICOS}

En CDB el grupo fitoplanctónico predominante fue el de los dinoflagelados (principalmente representados por Prorocentrum micans) y otros organismos microplanctónicos tales como criptófitas (representadas por el género Rhodomonas) y silicoflagelados (representados por el género Dictyocha) (Tabla 1). En superficie se observó poca variación en la densidad de fitoplancton de las 2 estaciones analizadas, mientras que a los $10 \mathrm{~m}$ de profundidad se detectó un incremento de la abundancia en la estación más cercana a la costa (Estación 4), en la cual se triplicaron los valores observados en superficie. La tasa de incorporación de nitrato correlacionó positiva y significativamente con la densidad del fitoplancton $($ Pearson $=0,99, P<0,05)$.

\section{Aguas costeras: Bahía Nueva (BN)}

\section{TASA DE INCORPORACIÓN DE NITRATO}

Entre la estación 1 de BN (5 km al sudeste de Puerto Madryn) y la estación 2 (frente a Puerto Madryn) la temperatura, salinidad, el $\mathrm{pH}$ y la turbidez de la columna de agua fueron similares desde la superficie al fondo (Fig. 3 ). Los valores medios observados fueron $16,3 \pm 0,15^{\circ} \mathrm{C}$; $33,86 \pm 0,05 ; 7,93 \pm 0,07$ y $7 \mathrm{~m}$, respectivamente. Los niveles de nitrato, fosfato y silicato también fueron similares en ambas estaciones y mostraron valores muy bajos $(0,19 \pm 0,12 \mu \mathrm{M} ; 0,85 \pm 0,09 \mu \mathrm{M}$ y $1,77 \pm 0,23 \mu \mathrm{M}$ respectivamente). A pesar de estas similitudes, la clorofila$a$ fue significativamente más elevada frente a la Ciudad que en la estación más alejada $(P<0,05)$. La tasa de incorporación de nitrato, en cambio, no presentó la misma tendencia (Fig 3). Ésta se caracterizó por diferencias significativas $(P<0,05)$ entre superficie $\left(43 \pm 0,3 \mathrm{nM} \mathrm{d}^{-1} \mathrm{y}\right.$ $\left.40 \pm 2,5 \mathrm{nM} \mathrm{d}^{-1}\right)$ y $3,5 \mathrm{~m}$ de profundidad $(13 \pm 8,0$ y $14 \pm 9,0$ 
Tabla 1. Abundancia (Cel. $\mathrm{L}^{-1}$ ) de los principales grupos fitoplanctónicos de las estaciones 2 y 4 a 0 y $10 \mathrm{~m}$ de profundidad en Cabo Dos Bahías / Abundance (Cel. $\left.\mathrm{L}^{-1}\right)$ of the main phytoplanktonic groups of the stations 2 and 4 at 0 and $10 \mathrm{~m}$ of depth in Cabo Dos Bahías

\begin{tabular}{lccccccc}
\hline Localidad & Estación & Profundidad & Diatomeas & Dinoflagelados & Otros & Nanoplancton & Total \\
\hline \multirow{2}{*}{ CDB } & 2 & 0 & 990 & 2580 & 1740 & 660 & 5970 \\
& 2 & 10 & 330 & 1300 & 580 & 330 & 2540 \\
& 4 & 0 & 1840 & 1800 & 1700 & 340 & 5680 \\
& 4 & 10 & 880 & 7040 & 11000 & 0 & 18920 \\
Total & & & 4040 & 12720 & 15020 & 1330 & 33110 \\
\hline
\end{tabular}

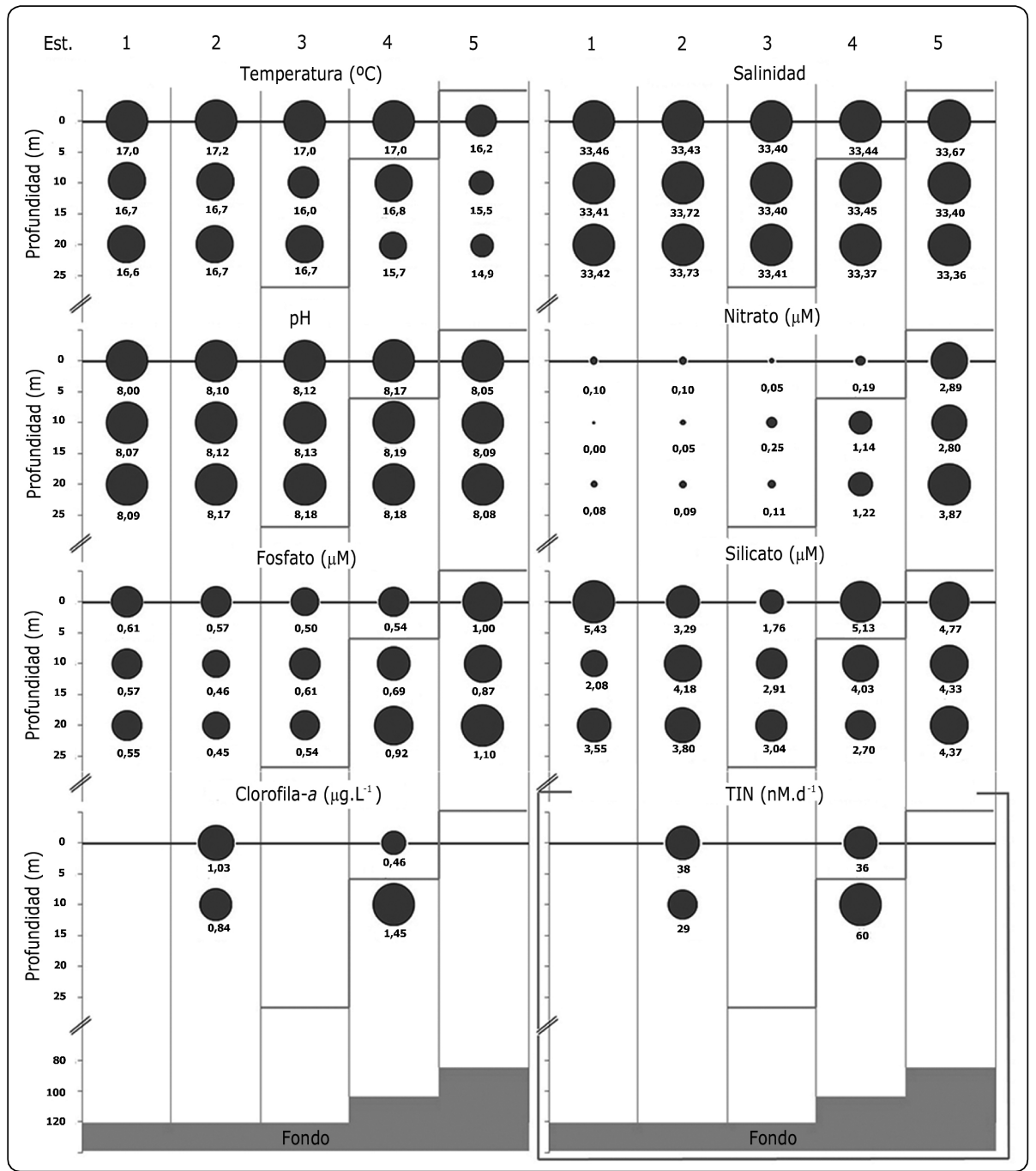

Figura 2. Distribución vertical de temperatura, salinidad, pH, nitrato, fosfato, silicato, clorofila-a e incorporación de nitrato por fitoplancton en cabo Dos Bahías. El tamaño de los círculos es proporcional a su valor para cada parámetro / Vertical distribution of temperature, salinity, $\mathrm{pH}$, nitrate, phosphate, silicate, chlorophyll-a and nitrate uptake by phytoplankton in cape Dos Bahías. The size of the circles is proportional to its value for each parameter 


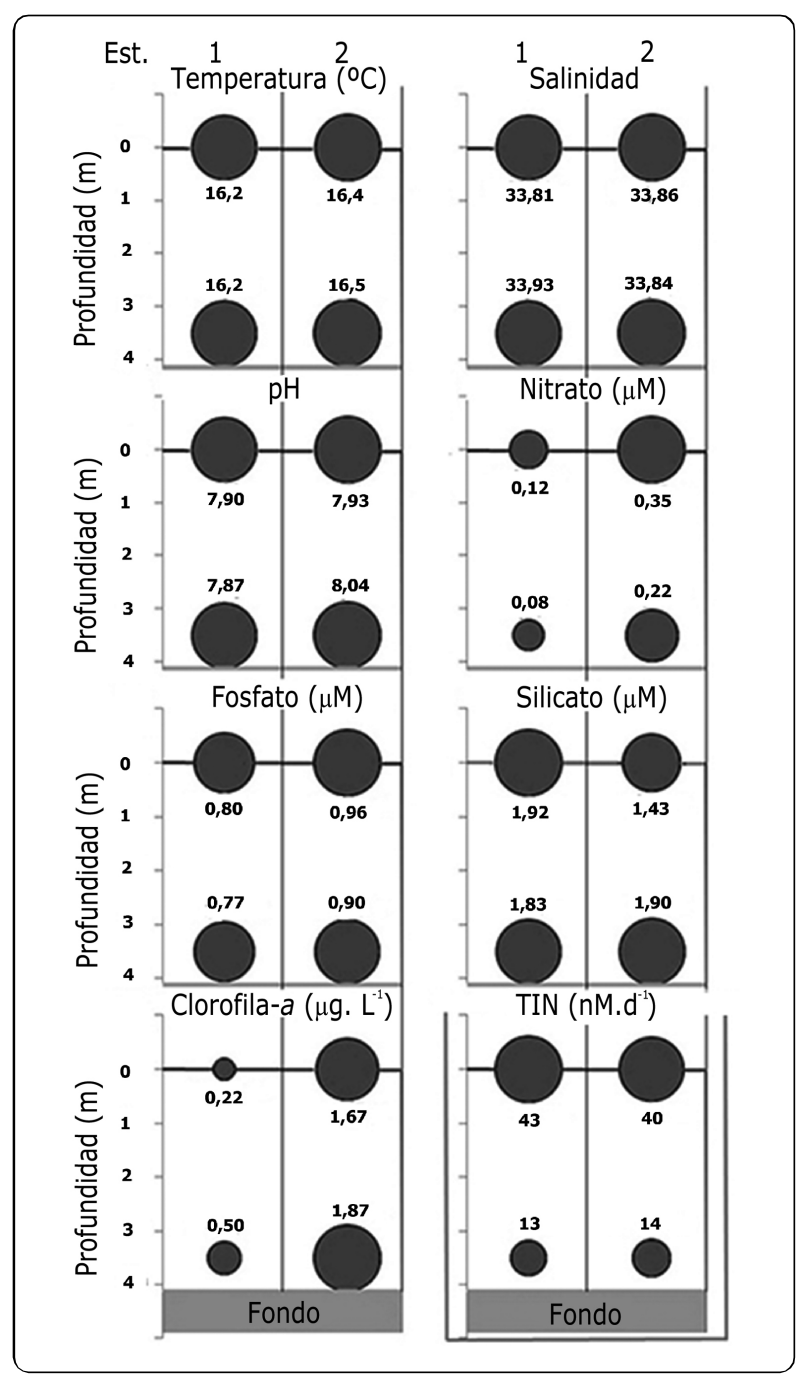

Figura 3. Distribución vertical de temperatura, salinidad, $\mathrm{pH}$, nitrato, fosfato, silicato, clorofila-a e incorporación de nitrato por fitoplancton en Bahía Nueva. El tamaño de los círculos es proporcional a su valor para cada parámetro / Vertical distribution of temperature, salinity, $\mathrm{pH}$, nitrate, phosphate, silicate, chlorophyll-a and nitrate uptake by phytoplankton in Bahía Nueva. The size of the circles is proportional to its value for each parameter

$\left.\mathrm{nM} \mathrm{d}^{-1}\right)$. El COP y el NOP fueron del orden de $150 \mu \mathrm{g} \mathrm{C} \mathrm{y}$ $27 \mu \mathrm{g} \mathrm{N}$, con valores mayores en la estación 1 respecto de la estación 2, y en superficie respecto del estrato más profundo investigado. La relación COP/NOP se mantuvo entre 6 y 6,7. La relación $\mathrm{NO}_{3} / \mathrm{PO}_{4}$ fue entre 0,1 y 0,$4 ; \mathrm{NO}_{3} /$ $\mathrm{SiO}_{3}$ entre 0,04 y 0,24 y $\mathrm{PO}_{4} / \mathrm{SiO}_{3}$ entre 0,41 y 0,67 .

\section{Proporción de grupos fitoplanctónicos}

En las estaciones de $\mathrm{BN}$ el grupo fitoplanctónico predominante fueron las diatomeas (principalmente representadas por el género Chaetoceros y en menor medida por Leptocylindrus y Thalassiosira) (Tabla 2). La abundancia fitoplanctónica no presentó diferencias en función de la profundidad en las 2 estaciones, aunque frente a la ciudad de Puerto Madryn la abundancia fue un $20 \%$ mayor que en la estación más apartada. Se observó una correlación positiva y significativa entre clorofila- $a$ y la abundancia total del fitoplancton (Pearson $=0,95, P<$ $0,05)$.

\section{Discusión}

\section{VARIACIONES AMbIENTALES ENTRE LAS ÁREAS BAJO ESTUDIO}

La BN se encuentra dentro del Golfo Nuevo, es un ambiente semicerrado con un intercambio muy reducido de aguas con el mar abierto (Rivas \& Beier 1990). Existen valores de nutrientes reportados previamente para el mismo sector y época del año (Esteves et al. 1981, 1992, Solís 1998) presentando concentraciones de nitrato muy fluctuantes, desde no detectables hasta $18 \mu \mathrm{M}$. Al respecto, altas concentraciones de amonio $(>2 \mu \mathrm{M})$ han sido asociadas al aporte antrópico (Gil 2001, Hasan 2003, Santinelli 2008) y concentraciones de fosfato entre 0,1 y 9,0 $\mu \mathrm{M}$ y de silicato entre 1,5 y 9,5 $\mu \mathrm{M}$ (Esteves et al. 1981, 1992, Sastre et al. 2001). Por el contrario, no existe información previa sobre las especies químicas bajo estudio en CDB. Allí, como fue expuesto en los resultados del presente trabajo, las estaciones más alejadas de la costa presentaron bajas concentraciones de nitrato y fosfato, incrementándose en las estaciones cercanas a la costa. Esta tendencia estuvo acompañada por la disminución de la temperatura.

En BN, el hecho de que la concentración de clorofila- $a$ fue significativamente más elevada en la estación situada frente a la Ciudad que en la más alejada, denota la influencia de un aporte antrópico. En CDB los nutrientes analizados presentaron valores intermedios en comparación con los máximos y mínimos mencionados en BN. Sin embargo, en CDB el incremento de nutrientes y clorofila- $a$ en la estación 4 a $10 \mathrm{~m}$ de profundidad y en los nutrientes de todas las profundidades de la estación 5 incentiva a realizar futuros estudios para investigar la posible presencia de un frente costero en dicho lugar.

La baja relación nitrato/fosfato en todas las estaciones analizadas, con valores del orden de 0,67 en CDB y 0,22 
Tabla 2. Abundancia (Cel. $\mathrm{L}^{-1}$ ) de los principales grupos fitoplanctónicos de las estaciones 1 y 2 a 0 y 3,5 m de profundidad en Bahía Nueva / Abundance ( $\left.\mathrm{Cel} . \mathrm{L}^{-1}\right)$ of the main phytoplanktonic groups of the stations 1 and 2 at 0 and $3.5 \mathrm{~m}$ of depth in Bahía Nueva

\begin{tabular}{lccccccc}
\hline Localidad & Estación & Profundidad(m) & Diatomeas & Dinoflagelados & Otros & Nanoplancton & Total \\
\hline BN & 1 & 0 & 11752 & 40680 & 6328 & 0 & 58760 \\
& 1 & 3,5 & 78196 & 2260 & 4520 & 0 & 84976 \\
& 2 & 0 & 91303 & 904 & 15368 & 0 & 107575 \\
& 2 & 3,5 & 100344 & 4068 & 6528 & 904 & 111844 \\
Total & & & 281595 & 47912 & 32744 & 904 & 363155 \\
\hline
\end{tabular}

en $\mathrm{BN}$, permite suponer una limitación general por nitrato en procesos de productividad primaria.

Con respecto a la tasa de incorporación de nitrato, los valores obtenidos en $\mathrm{BN}$ y $\mathrm{CDB}$ muestran similitud con aquellos observados en aguas oligotróficas, como las de Bermuda (Dugdale \& Goering 1967) o Groenlandia (Smith et al. 1997). Sólo en la estación 4 de CDB a $10 \mathrm{~m}$ de profundidad se registraron valores algo mayores, los cuales pueden estar relacionados a un frente costero. Fernández et al. (2009) obtuvieron resultados similares trabajando en un sector de surgencia del Mar de Perú. Por otro lado, y en coincidencia con otros estudios (Bode et al. 2005, Jang et al. 2008), en ambas estaciones de BN y en la estación 2 de CDB, la tasa de incorporación de nitrato siendo significativamente menor cerca del fondo que en superficie podría asociarse a la disminución de la intensidad lumínica.

Contrariamente a lo observado en algunos trabajos previos (Peña et al. 1992, McCarthy et al. 1996) la tasa de incorporación de nitrato no estuvo asociada a la concentración de clorofila- $a$. Esto podría deberse a la utilización de otras fuentes de nitrógeno por parte del fitoplancton. En BN, la baja concentración de nitrato en el medio podría favorecer la utilización de otra fuente nitrogenada, como se observó en otros trabajos (McCarthy 1972, McCarthy et al. 1977, Gilbert et al. 1982, Dortch 1990). Hasan (2003) detectó para esta zona un importante flujo de amonio asociado al agua subterránea. Además, han sido documentados incrementos significativos de la concentración de este nutriente debido a la eutrofización generada por el impacto de la ciudad de Puerto Madryn (Esteves et al. 1992, 1997, Gil 2001, Sastre et al. 2001, Mohamed 2008). Las concentraciones elevadas de amonio pueden inhibir la incorporación de nitrato por el fitoplancton (Murray et al. 1989, Price et al. 1994, Varela \& Harrison 1999). En presencia de bajas concentraciones de nitrato, el amonio puede representar cerca del $80 \%$ de la incorporación de nitrógeno (Dugdale \& Goering 1967, Mac Isaac \& Dugdale 1972, Fernández et al. 2009). En CDB no se cuenta con información sobre la concentración de amonio; sin embargo, por tratarse de un área abierta ubicada en la plataforma continental, se esperarían valores bajos de ese nutriente.

\section{RELACIÓN ENTRE PRODUCCIÓN NUEVA Y FITOPLANCTON}

Durante la cuantificación de la dominancia fitoplanctónica realizada en el presente trabajo, no se ha observado la presencia de diazótrofos. Esto concuerda con un estudio realizado en BN entre 1993-1998 (Santinelli 2008). En dicho estudio se destacó la baja abundancia de estos organismos en tales sectores marinos costeros. A partir de esto se asume que en el presente trabajo, mediante la medición de la incorporación de nitrato por el fitoplancton, se estaría estimando de manera precisa la producción nueva de las áreas bajo estudio.

En BN, las diatomeas constituyen la fracción más importante del microplancton durante el otoño, y los porcentajes de abundancia de dinoflagelados no superan el 20\% (Gayoso 2001, Santinelli 2008). Esto se debe a condiciones ambientales tales como la reducida intensidad del viento, elevada concentración de nutrientes, y una favorable profundidad de la capa de mezcla (Villafañe et al. 2004). Al igual que con el resto de los parámetros analizados, en CDB no existe información previa publicada sobre la estructura fitoplanctónica.

Según Bode et al. (2002), la producción nueva se asociaría al crecimiento inicial del fitoplancton 
(comunidades generalmente compuestas por diatomeas), mientras que la producción regenerada sería característica de etapas más avanzados de la sucesión (por ejemplo nanoflagelados) (Smith \& Nelson 1986, Goeyens et al. 1991). Sin embargo, en BN se observó una dominancia de diatomeas y en las estaciones cercanas al fondo la tasa de incorporación de nitrato fue una de las más bajas medidas en este experimento. Por el contrario, en CDB, en la estación 4, a $10 \mathrm{~m}$ de profundidad, se observó una concentración relativamente elevada de nutrientes y la máxima tasa de incorporación de nitrato, asociada a una dominancia de dinoflagelados y otros organismos tales como criptófitas. Los resultados del presente estudio son insuficientes para arribar a una conclusión similar a la propuesta por Bode et al. (2002). Sin embargo, la presencia de otras fuentes nitrogenadas en el medio puede haber sido causal directa de las variaciones observadas, principalmente en $\mathrm{BN}$ (donde, tal como se mencionó anteriormente, el amonio puede alcanzar concentraciones elevadas). Si se restringe la observación a las estaciones superficiales, en BN la incorporación de nitrato fue algo mayor que en CDB (aunque esta diferencia no fue estadísticamente significativa).

En CDB, la tasa de incorporación de nitrato correlacionó positiva y significativamente con la densidad del fitoplancton. En cambio, en BN estas variables no mostraron asociación. Los principales grupos fitoplanctónicos de ambas localidades fueron diferentes: diatomeas en $\mathrm{BN}$ y dinoflagelados y criptófitas en CDB. Esto deja abierta una pregunta: ¿será una regla general que en ambientes dominados por dinoflagelados y criptófitas la densidad fitoplanctónica este asociada a la producción nueva y en ambientes dominados por diatomeas esto no suceda?

En los 2 ambientes bajo estudio, la concentración de clorofila- $a$ no estuvo asociada a la producción nueva. Sin embargo en BN se observó una correlación positiva y significativa entre clorofila- $a$ y la abundancia total del fitoplancton. Esto, asociado a lo mencionado en el párrafo anterior, podría deberse a la estrecha relación entre ese pigmento y la abundancia de diatomeas (Villafañe et al. 2004).

En general, el experimento realizado en el presente estudio considera al fitoplancton como única fuente de incorporación de nitrato. Sin embargo, varios autores (Kirchman et al. 1991, Kroer et al. 1994, Kirchman \& Wheeler 1998, Allen et al. 2002) han demostrado que el nitrato sostiene además, para diversos ecosistemas marinos, el crecimiento de las bacterias heterótrofas. Por esta razón, y en coincidencia con lo propuesto por Rodrigues \& Williams (2002), las conclusiones obtenidas sobre la tasa de incorporación de nitrato pueden considerarse un estimador de la producción nueva generada por toda la comunidad de microrganismos, tanto autótrofos como heterótrofos.

A partir del primer paso alcanzado por el presente trabajo, se hace necesario incorporar la experimentación con sales de amonio y urea marcadas con ${ }^{15} \mathrm{~N}$ que permitan cuantificar la Producción Regenerada. La información obtenida de medir íntegramente la incorporación de compuestos nitrogenados por el fitoplancton será de gran importancia para la comprensión del funcionamiento de estos ecosistemas.

\section{Agradecimientos}

Se agradece al personal del Laboratorio de Oceanografía Química y Contaminación de Aguas del Centro Nacional Patagónico, en especial a la MSc. Miriam Solís por su colaboración durante los análisis de laboratorio. A la Fundación Patagonia Natural y al Sr. Lorenzo Giménez por haber facilitado las embarcaciones necesarias para la toma de muestras en Bahía Nueva y en cabo Dos Bahías respectivamente. A la Lic. Viviana Sastre por su colaboración durante el recuento de fitoplancton. Finalmente, se agradece encarecidamente a los evaluadores por su dedicación y por ayudar a mejorar el presente trabajo. El estudio fue financiado por CONICET PIP 5507, y PICTO 20100128 (otorgado a VAA).

\section{LiTERATURA CITADA}

Allen AE, MH Howard-Jones, MG Booth, ME Frischer, PG Verity, DA Bronk \& MP Sanderson. 2002. Importance of heterotrophic bacterial assimilation of ammonium and nitrate in the Barents Sea during summer. Journal of Marine Systems 38: 93-108.

Bode A, CG Castro, MD Doval \& M Varela. 2002. New and regenerated production and ammonium regeneration in the western Bransfield Strait region (Antarctica) during phytoplankton bloom conditions in summer. Deep-Sea Research II 49: 787-804.

Bode A, N Gonzalez, C Rodriguez, M Varela \& MM Varela. 2005. Seasonal variability of plankton blooms in the Ria de Ferrol (NW Spain): I. Nutrient concentrations and nitrogen uptake rates. Estuarine, Coastal and Shelf Science 63: 269284.

Brandhorst W \& JP Castello. 1971. Evaluación de los recursos de anchoíta (Engraulis anchoita) frente a la Argentina y Uruguay. I. Las condiciones oceanográficas, sinopsis del 
conocimiento actual sobre la anchoíta y el plan para su evaluación. Contribuciones del Instituto de Biología Marina, Mar del Plata, Argentina 166: 1-63.

Cabrita MT, F Catarino \& G Slawyk. 1999. Interactions of light, temperature and inorganic nitrogen in controlling plankton nitrogen utilisation in the Tagus estuary. Aquatic Ecology 33: 251-261.

Carreto JI, VA Lutz, MO Carignan, AD Cucchi-Colleoni \& SG De Marco. 1995. Hydrography and chlorophyll- $a$ in a transect from the coast to the shelf-break in the Argentinian Sea. Continental Shelf Research 15(2/3): 315-336.

Carreto JI, MO Carignan, NG Montoya \& AD CucchiColleoni. 2007. Ecología del fitoplancton en los sistemas frontales del Mar Argentino. En: Carreto JI \& C Bremec (eds). El mar argentino y sus recursos pesqueros 5: 11-13. INIDEP, Mar del Plata.

Dortch Q. 1990. The interaction between ammonium and nitrate uptake in phytoplankton. Marine Ecology Progress Series 61: 183-201.

Dugdale RC \& JJ Goering. 1967. Uptake of new and regenerated forms of nitrogen in primary productivity. Limnology and Oceanography 12(2): 196-206.

Esteves JL, H De Vido, N de Mattio, JJ Cejas \& J Frontali. 1981. Evolución de parámetros químicos y biológicos en el área de Bahía Nueva (Golfo Nuevo). Contribución, Centro Nacional Patagónico 57: 1-25.

Esteves JL, N Santinelli, V Sastre, R Díaz \& O Rivas. 1992. A toxic dinoflagellate bloom and P.S.P. production associated with upwelling in Golfo Nuevo, Patagonia, Argentina. Hydrobiologia 242: 115-122.

Esteves JL, M Solís, MN Gil, N Santinelli, V Sastre, C González-Raies, M Hoffmeyer \& M Commendatore. 1997. Evaluación de la contaminación urbana de la Bahía Nueva (provincia de Chubut). Informes Técnicos del Plan de Manejo Integrado de la Zona Costera Patagónica (Puerto Madryn, Argentina) 31: 1-35.

Fernández C, L Farías \& ME Alcaman. 2009. Primary production and nitrogen regeneration processes in surface waters of the Peruvian upwelling system. Progress in Oceanography 83: 159-168.

Fernández C, L Farías \& O Ulloa. 2011. Nitrogen fixation in denitrified marine waters. PLoS ONE 6(6): e20539.

Gayoso AM. 2001. Observations on Alexandrium tamarense (Lebour) Balech and other dinoflagellate populations in Golfo Nuevo, Patagonia (Argentina). Journal of Plankton Research 23: 463-468.

Gil MN. 2001. Eutroficación: Rol del nitrógeno en ecosistemas marinos costeros. Tesis Doctorado Ingeniería Química, Universidad Nacional del Sur, Bahía Blanca, 127 pp.

Gilbert PM, DC Biggs \& JJ McCarthy. 1982. Utilization of ammonium and nitrate during austral summer in the Scotia Sea. Deep Sea Research I 29, 837-850.
Goeyens L, F Sorensson, P Treguer, J Morvan, M Panouse \& F Dehairs. 1991. Spatiotemporal variability of inorganic nitrogen stocks and uptake fluxes in the Scotia-Weddell Confluence area during November and December 1988. Marine Ecology Progress Series 77: 7-19.

Hasan EJ. 2003. Caracterización de sedimentos infralitorales a través del intercambio de nutrientes de la interfase aguasedimento de la Bahía Nueva (Golfo Nuevo, Chubut, Argentina). Tesis de Licenciatura, Universidad Nacional de la Patagonia San Juan Bosco, Puerto Madryn, 65 pp.

Jang YH, BK Khim, HC Shin, DM Sigman, Y Wang \& CS Hong. 2008. Variation of nitrate concentrations and $\delta{ }^{15} \mathrm{~N}$ values of seawater in the Drake Passage, Antarctic Ocean. Ocean and Polar Research 30(4): 407-418.

Kirchman DL \& PA Wheeler. 1998. Uptake of ammonium and nitrate by heterotrophic bacteria and phytoplankton in the sub-Arctic Pacific. Deep-Sea Research I 45: 347-365.

Kirchman DL, Y Suzuki, C Garside \& HW Ducklow. 1991. High turnover rates of dissolved organic carbon during a spring phytoplankton bloom. Nature 352: 612-614.

Kroer N, NOG Jørgensen \& RB Coffin. 1994. Utilization of dissolved nitrogen by heterotrophic bacterioplankton: a comparison of three ecosystems. Applied and Environmental Microbiology 60: 4116-4123.

Lund JWG, C Kilpling \& ED Le Cren. 1958. The inverted microscope method of estimating algal numbers, and the statistical basis of estimation by counting. Hydrobiologia 11(2): 143-170.

Mac Isaac JJ \& RC Dugdale. 1972. Interactions of light and inorganic nitrogen in controlling nitrogen uptake in the sea. Deep-Sea Research I 19: 209-232.

McCarthy JJ. 1972. The uptake of urea by natural populations of marine phytoplankton. Limnology and Oceanography 17: 738-748.

McCarthy JJ, WR Taylor \& JL Taft. 1977. Nitrogenous nutrition of the plankton in the Cheaspeake Bay, Nutrient availability and phytoplankton preferences. Limnology and Oceanography 22: 996-1011.

McCarthy JJ, C Garside, JL Nevins \& RT Barber. 1996. New production along $140^{\circ} \mathrm{W}$ in the equatorial Pacific during and following the 1992 El Niño event. Deep Sea Research II 43: 1065-1093.

Mohamed NN. 2008. Evaluación de la concentración de metales pesados en moluscos bivalvos, material particulado y sedimentos de la zona costera del Chubut. Seminario de Licenciatura en Ciencias Biológicas, Facultad de Ciencias Naturales, Universidad Nacional de la Patagonia, Ciudad de Puerto Madryn, 114 pp.

Montoya JP, CM Holl, JP Zehr, A Hansen, TA Villareal \& DG Capone. 2004. High rates of $\mathrm{N} 2$ fixation by unicellular diazotrophs in the oligotrophic Pacific Ocean. Nature 430: 1027-1031. 
Murray JW, JN Downs, S Strom, CL Wei \& HW Jannasch. 1989. Nutrient assimilation, export production and $234 \mathrm{Th}$ scavenging in the eastern equatorial Pacific. Deep Sea Research I 36: 1471-1489.

Paparazzo FE. 2003. Evolución de nutrientes inorgánicos en aguas oceánicas y su relación con la biomasa fitoplanctónica. Seminario de Licenciatura en Ciencias Biológicas, Universidad Nacional de la Patagonia San Juan Bosco, Puerto Madryn, Chubut, 90 pp.

Paparazzo FE, L Bianucci, IR Schloss, GO Almandoz, M Solís \& JL Esteves. 2010. Cross-frontal distribution of inorganic nutrients and chlorophyll- $a$ on the Patagonian Continental Shelf of Argentina during summer and fall. Revista de Biología Marina y Oceanografía 45(1): 107-119.

Peña MA, WG Harrison \& MR Lewis. 1992. New production in the central equatorial Pacific. Marine Ecology Progress Series 8: 265-274.

Price NM, BA Ahner \& FMM Morel. 1994. The equatorial Pacific Ocean: Grazer-controlled phytoplankton populations in an iron-limited ecosystem. Limnology and Oceanography 39: 520-534.

Rodrigues RM, NV Williams \& Le BPJ. 2002. Inorganic nitrogen assimilation by picoplankton and whole plankton in a coastal ecosystem. Limnology and Oceanography 47: 1608-1616.

Santinelli NH. 2008. Fitoplancton de un ambiente costero sometido a perturbación antrópica: Bahía Nueva, Provincia de Chubut. Tesis doctoral, Facultad de Ciencias Naturales, Universidad Nacional de la Patagonia San Juan Bosco, Trelew, 216 pp.

Sastre AV, NH Santinelli, JL Esteves \& ME Ferrario. 2001. Aspectos ecológicos de especies de Pseudo-nitzschia en aguas costeras patagónicas (Argentina). En: Alveal K \& T Antezana (eds). Sustentabilidad de la biodiversidad: un problema actual, bases científico-técnicas, teorizaciones y proyecciones, pp. 217-235. Universidad de Concepción, Concepción.
Smith WO \& DM Nelson. 1986. Importance of the ice edge phytoplankton production in the Southern Ocean. Bioscience 36 (4): 251-257.

Smith W Jr, M Gosselin, L Legendre, D Wallace, K Daly \& G Kattner. 1997. New production in the Northeast Water Polynya: 1993. Journal of Marine Systems 10: 199-209.

Solís M. 1998. Monitoring in Nuevo Gulf (Argentina): Analysis of oceanographic data by geographic information systems (GIS). MSc. Thesis (D.E.W. 021-,IHE), UNESCO IHE, Institute for Water Education, Delft, 140 pp.

Strickland JDH \& TR Parsons. 1972. A practical handbook of seawater analysis. Fisheries Researches Board of Canada, Bulletin 167: 1-310.

Technicon. 1977. Technicon Ind. Method 186-72/W: Silicate in water and seawater, 10591 pp. Technicon Ind. Systems, Tarrytown.

Varela DE \& PH Harrison. 1999. Seasonal variability in nitrogenous nutrition of phytoplankton assemblages in the northeastern subarctic Pacific Ocean. Deep-Sea Research II 46: 2505-2538.

Villafañe VE \& FM Reid. 1995. Métodos de microscopía para la cuantificación del fitoplancton. En: Alveal K, ME Ferrario, EC Oliviera \& E Zar (eds). Manual de métodos ficológicos, pp. 170-183. Universidad de Concepción, Concepción.

Villafañe VE, MA Marcoval \& WE Helbling. 2004. Photosynthesis versus irradiance characteristics in phytoplankton assemblages off Patagonia (Argentina): temporal variability and solar UVR effects. Marine Ecology Progress Series 284: 23-34.

Recibido el 3 de noviembre de 2012 y aceptado el 18 de octubre de 2013

Editor: Claudia Bustos D. 